\title{
Cell adhesion property affected by cyclooxygenase and lipoxygenase: Opto-electric approach
}

\author{
Chang Kyoung Choi ${ }^{a}$, Mugdha Sukhthankar ${ }^{b}$, Chul-Ho Kim $^{b}$, Seong-Ho Lee ${ }^{\mathrm{b}}$, Anthony English ${ }^{\mathrm{c}}$, \\ Kenneth D. Kihm ${ }^{c}$, Seung Joon Baek ${ }^{\text {b,* }}$ \\ ${ }^{a}$ Department of Mechanical Engineering - Engineering Mechanics, Michigan Technological University, Houghton, MI 49931, USA \\ ${ }^{\mathrm{b}}$ Department of Pathobiology, College of Veterinary Medicine, University of Tennessee, Knoxville, TN 37996, USA \\ ${ }^{\mathrm{c}}$ Department of Mechanical, Aerospace, and Biomedical Engineering, University of Tennessee, Knoxville, TN 37996, USA
}

\section{A R T I C L E I N F O}

\section{Article history:}

Received 20 November 2009

Available online 22 December 2009

\section{Keywords:}

Cyclooxygenase

DICM

IRCM

Lipoxygenase

Impedance

\begin{abstract}
A B S T R A C T
Expression of cyclooxygenases (COX) and lipoxygenases (LOX) has been linked to many pathophysiological phenotypes, including cell adhesion. However, many current approaches to measure cellular changes are performed only in a fixed-time point. Since cells dynamically move in conjunction with the cell matrix, there is a pressing need for dynamic or time-dependent methods for the investigation of cell properties. In the presented study, we used stable human colorectal cancer cell lines ectopically expressing COX-1, COX-2, and 15LOX-1, to investigate whether expression of COX-1, COX-2, or 15LOX-1 would affect cell adhesion using our opto-electric methodology. In a fixed-time point experiment, only COX-1and COX-2-expressing cells enhanced phosphorylation of focal adhesion kinase, but all the transfected cells showed invasion activity. However, in a real-time experiment using opto-electric approaches, transmitted cellular morphology was much different with tight adhesion being shown in COX-2 expressing cells, as imaged by differential interference contrast microscopy (DICM) and interference reflection contrast microscopy (IRCM). Furthermore, micro-impedance measurements showed a continued increase in both resistance and reactance of COX- and LOX-transfected cells, consistent with the imaging data. Our data indicate that both COX- and LOX-expressing cells have strong cell-to-cell and cell-to-substrate adhesions, and that cell imaging analysis with cell impedance data generates fully reliable results on cell adhesion measurement.
\end{abstract}

(c) 2009 Elsevier Inc. All rights reserved.

\section{Introduction}

Cyclooxygenase (COX) and lipoxygenase (LOX) have been associated with tumorigenesis in several cancers [1], and their inhibition is desirable for cancer prevention. Major metabolites of COX and LOX, prostaglandin E2 and 15(S)-HETE, respectively, activate specific growth factor receptor-related signaling pathways, thereby initiating signal transduction events leading to increased cell adhesion to the extracellular matrix (ECM) [2]. Thus, it stands to reason that over-expression of COX-2 leads to phenotypic changes involving increased adhesion to the ECM [3], and 12/15LOX pathways increase cell adhesion molecules mediated by NF$\kappa \mathrm{B}$ activation [4]. Both COX and LOX may play an important role in cell adhesion.

Cell adhesion encompasses complex mechanisms involved in a variety of processes, including migration, invasion, wound healing

\footnotetext{
* Corresponding author. Address: Department of Pathobiology, College of Veterinary Medicine, University of Tennessee, 2407 River Drive, Knoxville, TN 37996, USA. Fax: +1 8659745616.

E-mail address: sbaek2@utk.edu (S.J. Baek).
}

and tissue remodeling. Adherent cells, such as those found in epithelial tissues like the colon, must be physically associated with an ECM to survive. During tumor formation, invasion, and metastasis, regulation of cell-ECM interactions is altered or lost. Cell adhesion to the ECM involves integrin binding, receptor clustering, and recruitment of cytoskeletal elements, leading to the formation of discrete adhesive structures (focal adhesions). Focal adhesion kinase (FAK) plays an important role in focal adhesion, mediated by phosphorylation; dysregulation of FAK signaling is implicated in the malignant transformation of cells, as well as in non-malignant pathological conditions. It has been reported that focal adhesion assembly enhanced adhesion strength by $30 \%$ over integrin clustering alone [5]. Although no direct linkage between the 15LOX-1 pathway and FAK has been reported, FAK signaling connections to COX-1 [6] and COX-2 [7] reflect tumorigenesis that may contribute to angiogenesis.

Many biologists have examined the molecular mechanisms of cell adhesion; however, the vast majority of these studies are based on static samples taken at a fixed-time point using conventional biological methodology. Cells are dynamically changing and constantly moving and interacting with other cells. Therefore, it 
is essential for dynamic or time-dependent investigation of cellcell and cell-substrate communication to be developed. Furthermore, quantitative and comprehensive assessment of cell adhesion and associated protein movements is desirable in order to evaluate the use of anti-cancer compounds for several cancers.

Several well-known methods used to examine cell adhesion and to provide bio-physiological and cellular function information on the effects of proteins and molecules of interest include fluorescence microscopy, flow cytometry, and biochemical assays. These methods are stationary and require staining with fluorescent or radioactive probes. In contrast, the proposed micro-impedance measurement in parallel with microscopic imaging techniques is a new bio-analytical method capable of non-invasively and dynamically monitoring cell adhesion in real-time. Another benefit is its high sensitivity to detect cellular micro-motions and the physiological changes of cells and cellular environments. Thus, this kind of an integrated dynamic bioelectrical impedance analysis along with microscopic live cellular imaging led us to evaluate cell physiology in a simpler and more effective way. In addition to cellular adhesion measurement, the micro-impedance system has been used to examine cellular proliferation [8], cytotoxicity [9], apoptosis [10], and cellular transformation [11]. In this study, we report our evaluation of opto-electro measurements of cell adhesion, affected by enzymes that are involved in arachidonic pathways. Cell imaging with cell impedance experiments represent the real-time profile of cellular dynamic adhesion activity and provides us quantitative analysis on cell adhesion activity.

\section{Materials and methods}

Cells and antibodies. For this study, HCT-116 cells serve as the negative control cells with transfected empty vectors because they do not constitutively express 15-LOX1, COX-1, and COX-2. Over-expressed stable cell lines are prepared to examine adhesion effects of these enzymes. HCT-116 human colorectal adenocarcinoma cells (ATCC, Manassas, VA) were maintained in McCoy 5A culture medium supplemented with $10 \%$ fetal bovine serum, $100 \mathrm{U}$ penicillin and $100 \mu \mathrm{g} / \mathrm{mL}$ streptomycin. The cells were grown at $37{ }^{\circ} \mathrm{C}$ under $5 \% \mathrm{CO}_{2}$. Stable cell lines in this study were previously reported $[12,13]$. Antibodies were purchased from Cell signaling (total FAK and phosphor-FAK), Oxford Biomedical Research Inc., (15-LOX1), Cayman Chemicals, (COX-1) and Santa Cruz Biotech., (COX-2 and Actin).

Micro-impedance measurement. LabVIEW (National Instruments Corp., Austin, Texas) was used to implement a data acquisition and analysis system. A reference voltage source of an ac $1 V_{\text {rms }}$ was provided via a series $1 \mathrm{M} \Omega$ resistor, $R_{\mathrm{cc}}$, to a gold electrode array (Applied Biophysics, Troy, NY). A National Instruments SCXI-1331 switch made successive connections between the live working electrodes and the counter electrode of each array. The source voltage generator resistance, $R_{\mathrm{s}}$, was $50 \Omega$. An SR830 lock-in amplifier (Stanford Research, Sunnyvale, CA) with an input impedance equivalent to a parallel resistor, $R_{\mathrm{v}}$, and capacitor, $C_{\mathrm{v}}$, combination of $10 \mathrm{M} \Omega$ and $10 \mathrm{pF}$, respectively, measured the electrode voltage. Direct measurements of the cable parasitic capacitances were made using an LCR meter and incorporated into a circuit model to estimate the impedance based on the lock-in amplifier voltage measurements. Any debris on the electrodes as well as electrode defects were examined by performing the preliminary naked scan. A second naked scan, sampled at a rate of $32 \mathrm{~Hz}$, was then performed for the naked electrodes. The electrodes were then inoculated with $400 \mu \mathrm{L}$ medium containing HCT-116 cells having a concentration of $10^{5}$ cells/ $\mathrm{mL}$. During the cellular attach scan, data was acquired at a rate of $32 \mathrm{~Hz}$ for $2 \mathrm{~s}$ using a $30 \mathrm{~ms}$ filter time constant and a $12 \mathrm{~dB} /$ decade roll off. Averages and standard deviation estimates were obtained from the sampled data points. During the experiments, cell-inoculated electrodes were kept in a cell culture incubator that maintained the temperature at $37^{\circ} \mathrm{C}$ and the $\mathrm{CO}_{2}$ level at $5 \%$.

Optical imaging system. A Xenon light source/IRCM illuminator attached to an Olympus IX-71 inverted microscope was used to create the natural fringe patterns for imaging the ventral surface of a cell. The fringe patterns of IRCM images are created by the interference of the two reflected rays, one ray from the cover slip-medium interface and the other ray from the medium-cell interface. A plan apochromat $100 \times$ oil immersion objective with adjustable numerical aperture (NA) was used for all imaging, and all images were captured using a 14-bit electron multiplier charge coupled device (EMCCD, Hamamatsu Corp.) A Halogen light source, plane polarizer, condenser Nomarski prism, objective Nomarski prism, and an analyzer were used for examining the whole cell including the dorsal surface, by way of DICM. It should be noted that an incubator was attached to the microscope, which provided the same temperature and percent $\mathrm{CO}_{2}$ used for maintaining and culturing cells. In order to effectively control the illuminated NA, our aperture iris was set to fully open, and the NA of the objective lens was set at 1.15. It was observed that an NA of 1.3 was too bright and provided a very shallow depth of focus (DOF), while setting the NA below 1.0 obscured fringes because of multiple interferences reflected from the deeper region. For excessive DOF created by a small NA, the possibility of additional reflections from the nucleus and/or dorsal membrane can bias the cell-substrate gap information. In addition, the large DOF made it inevitable that higher-order fringes would obscure the fringe analysis. The substantially reduced DOF for the higher NA prevented the multiple reflections and eliminated higher-order fringes. Therefore, the use of a high NA objective retained only the zero-order fringes, allowing monotonic intensity variations correlating with the gap distance between the cell and substrate.

Invasion assay. The matrigel invasion assays were performed using Transwell chambers (Costar, Corning, NY). Filters (pore size $8 \mu \mathrm{m}$ ) coated with matrigel (BD Bioscience, Bedford, MA) in the upper compartment were loaded with $100 \mu \mathrm{L}$ of McCoy $5 \mathrm{~A}$ media containing $1 \times 10^{6}$ cells, and the lower compartment was filled with McCoy $5 \mathrm{~A}$ and supplemented with $50 \mathrm{ng} / \mathrm{mL}$ insulin growth factor. The chamber was then cultivated in $5 \% \mathrm{CO}_{2}$ at $37{ }^{\circ} \mathrm{C}$ for $48 \mathrm{~h}$. The matrigel and cells on the filter were removed, and the attached cells in the lower section were stained with hematoxylin and eosin. These cells were counted and photographed under a light microscope.

Western blot analysis. Stable cell lines over-expressing 15-LOX1, COX-1, COX-2 and empty vector were grown to $60-80 \%$ confluence in 6 -cm plates for $24 \mathrm{~h}$. Total cell lysates were isolated using RIPA buffer ( $1 \times$ PBS, $1 \%$ NP-40, $0.5 \%$ sodium deoxycholate, $0.1 \%$ SDS). Proteins $(100 \mu \mathrm{g})$ were separated by SDS-PAGE and transferred for $1 \mathrm{~h}$ onto nitrocellulose membrane (Osmonics Inc., Minnetonka, MN). The blots were blocked for $1 \mathrm{~h}$ with $5 \%$ skim milk in TBS/ Tween $0.05 \%$ (TBS-T), and probed with FAK and phosphor-FAK antibodies (Cell signaling, Beverly, MA), 15-LOX1 (Oxford Biomedical research Inc., Oxford, MI), COX-1 (Cayman, MI), and COX-2 (Santa Cruz Biotech., Santa Cruz, CA) at $4{ }^{\circ} \mathrm{C}$ overnight. After washing with TBS-T, the blots were treated with horseradish peroxidase-conjugated secondary antibody for $1 \mathrm{~h}$ and washed several times. Proteins were detected by the enhanced chemiluminescence system (Amersham, IL) using Fujifilm developer.

\section{Results and discussion}

\section{Optical cellular imaging and corresponding optical results}

Time lapse optical images were examined to see if empty vector, 15LOX-1, COX-1, and COX-2 transfected cells showed different 
morphological changes. IRCM was used to map the dynamic fingerprint of cells' ventral surface and to examine the bottom morphology as a function of time, in particular, after the confluence condition. In addition, DICM sequentially imaged the entire cells' morphological changes as well as the evolution of live cells (Fig. 1). It was qualitatively observed that COX-2-transfected HCT-116 cells seem to have the strongest intercellular contacts, as they look more tightly packed, shown in DICM images, in comparison with other cell lines, and also have strong cell-substrate adhesion, as they look flatter in DICM images and have more dark areas in IRCM images (Fig. 1A and B). At 4.5 days, all the transfected cells showed a similar pattern in IRCM images. It should be noted that no clear piling of HCT-116 cells was observed. However, a few cells transfected with empty vector, 15LOX-1, and COX-1 in the field of view had relatively round shapes and did not touch the substrate. But COX-2 over-expressing cells had well-arranged cell attachment and uniform cellular thickness, which can be examined by changing focal planes from the bottom to the top of cells. It is not believed these cell lines grow as multi-layers.

Subsequently, we utilized digital imaging processing of the corresponding IRCM images. Graphs shown in Fig. 1C present the total number of pixels in terms of pixel grey levels (PGLs) grouped into intensity bins of 1000 for IRCM images. Note that cells reached confluency approximately 2 days after inoculation, and darker PGL values represent the smaller gaps between cell and substrate, i.e., the smallest gap is known to be the focal contacts. As shown in Fig. 1C, both COXs and 15LOX-1-transfected cells have darker PGLs than control cells, indicating that the former cells have smaller gaps between cells and substrate than the latter at 3.0 days. The total number of pixels shifted to the darker PGL in all the transfected

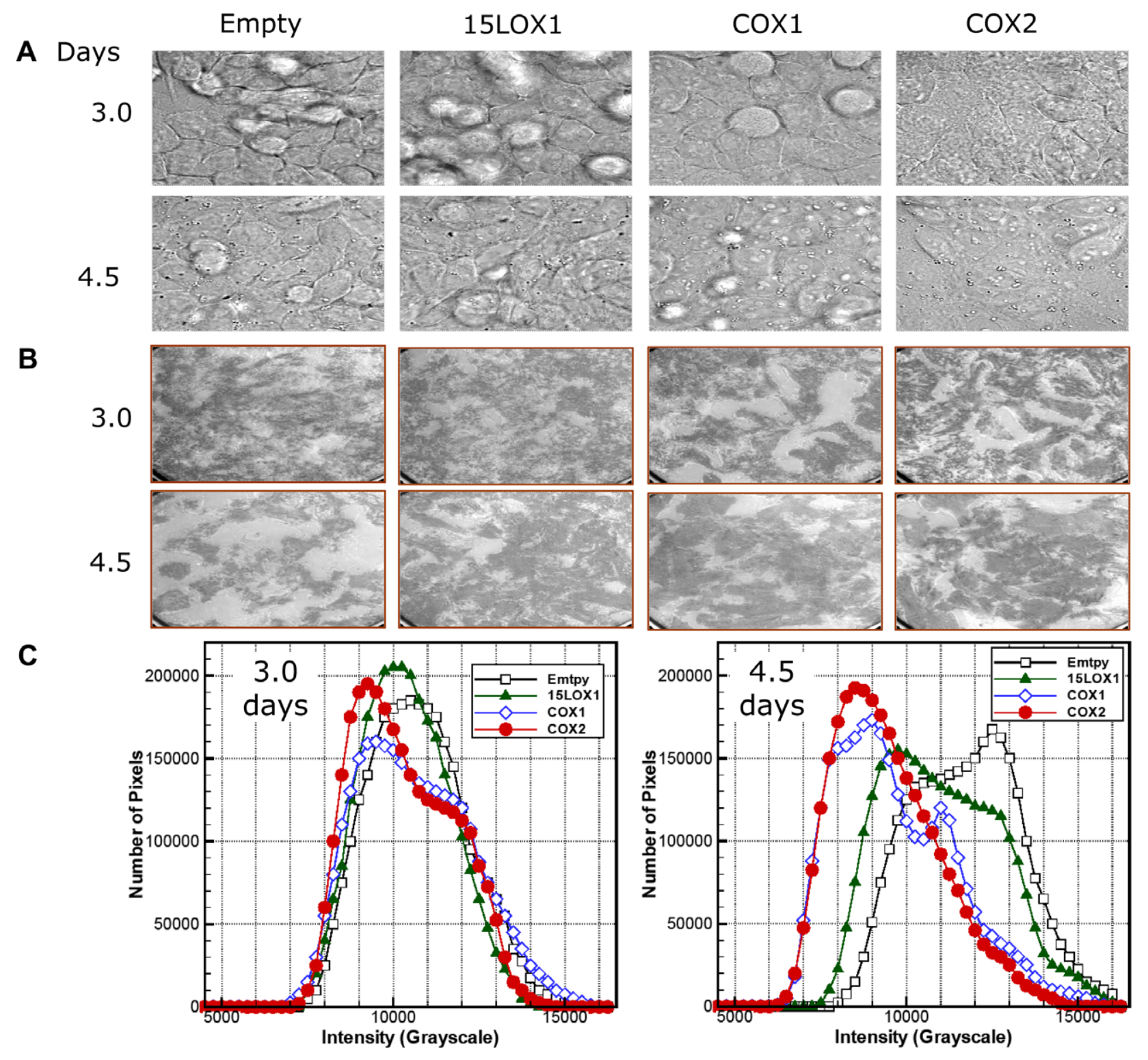

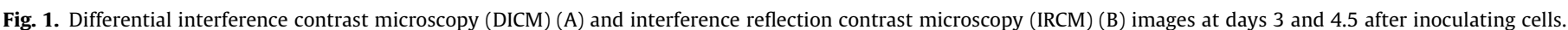

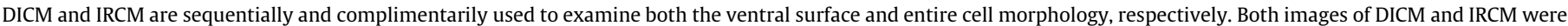

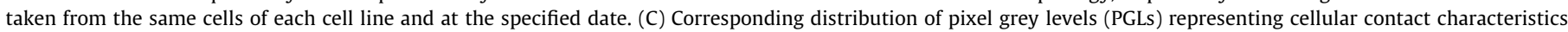

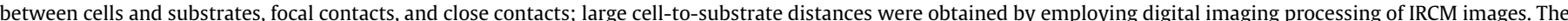

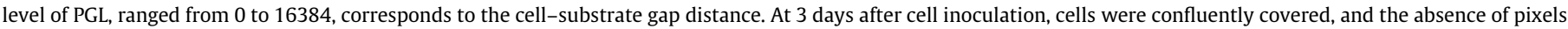
represents an area that is not occupied by cells. 
HCT-116 cells peaked at 4.5 days, becoming a more obviously tight-knit interaction than control cells, as seen in the IRCM images shown in Fig. 1B.

Interestingly, until 3 days after inoculating cells, there is no significant difference in the pixel intensity distribution of IRCM images representing bottom of the cells. However, at 4.5 days, the number of pixels of lower intensity representing focal contact, the closest distance between cells and substrates, increased for cells transfected with COX-2 and COX-1. Conversely, those of higher intensity increased for HCT-116 cells transfected with empty vector and 15-LOX1, indicating that there is weak contact between these cells and their substrate.

\section{Resistance and reactance}

Micro-impedance measurement has been widely used to dynamically examine micro-motion, proliferation, cell adhesion (cell-to-cell and cell-to-extracellular matrix), apoptosis, and cytotoxicity $[8-10,14]$. As shown in Fig. 2, the resistance and reactance micro-impedance measurements were carried out in parallel with the optical imaging experiment (Fig. 1). Although resistance measurements initially did not show a marked difference until 2 days after cell inoculation, the resistance of empty vector and LOXtransfected HCT- 116 cells plateaued, while that of COX-transfected cells continued increasing for the remainder of the experiment.
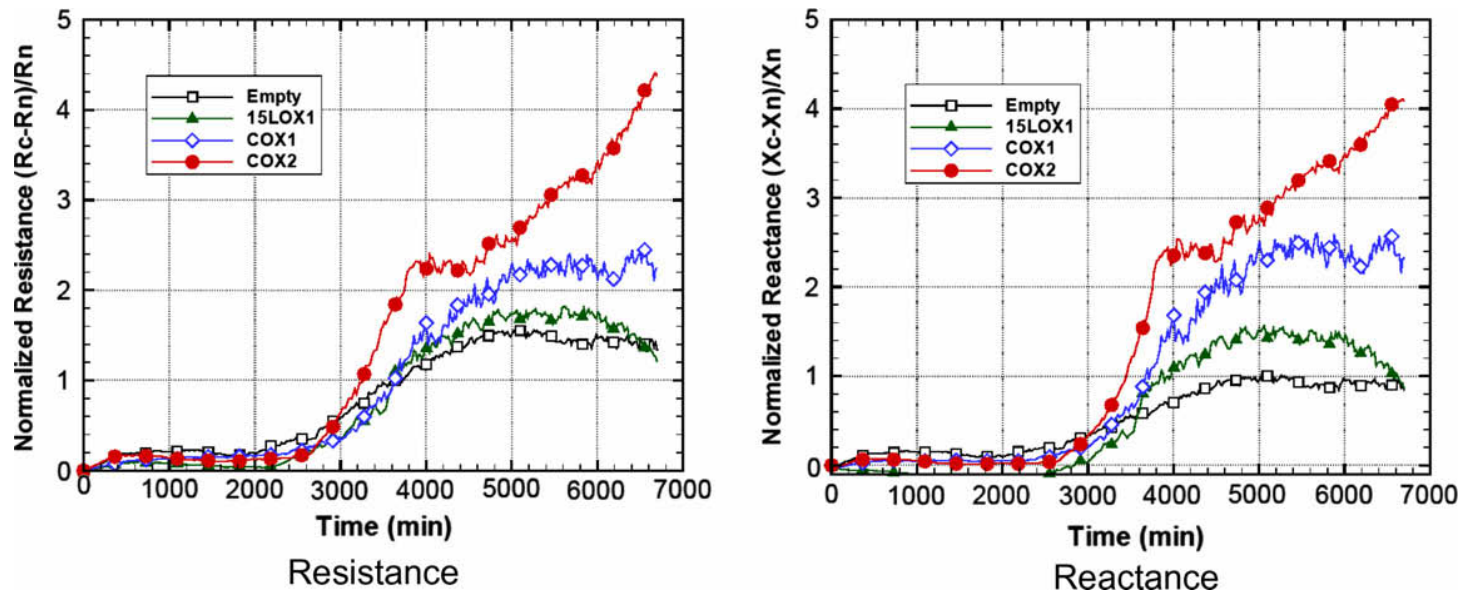

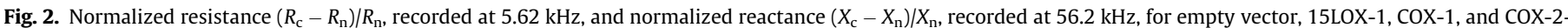

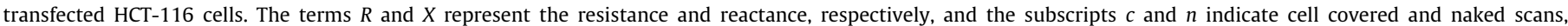
respectively.

A
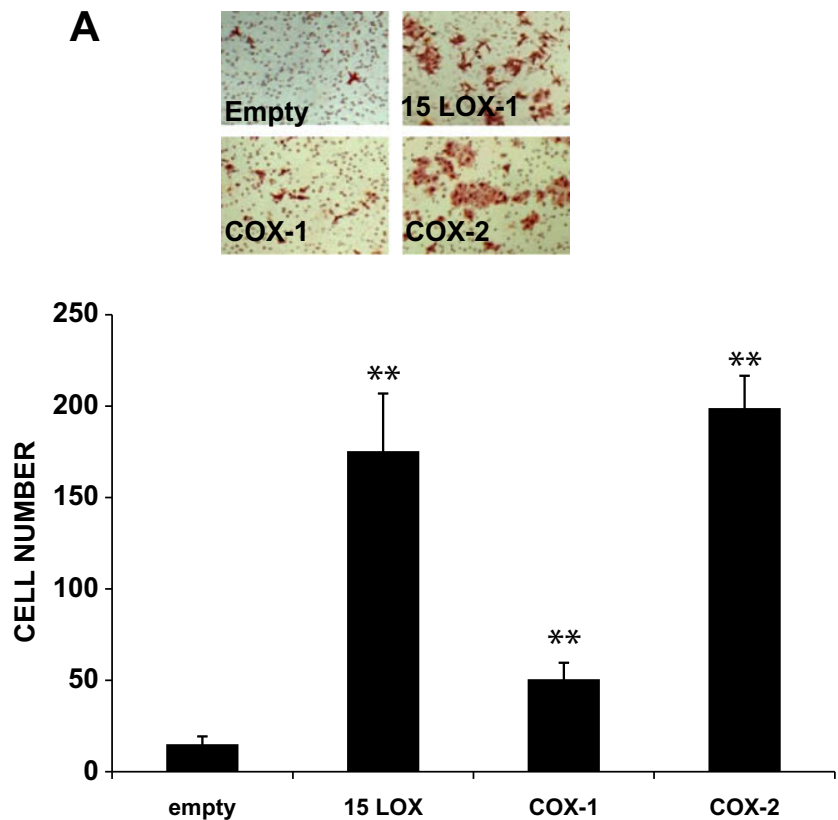

B

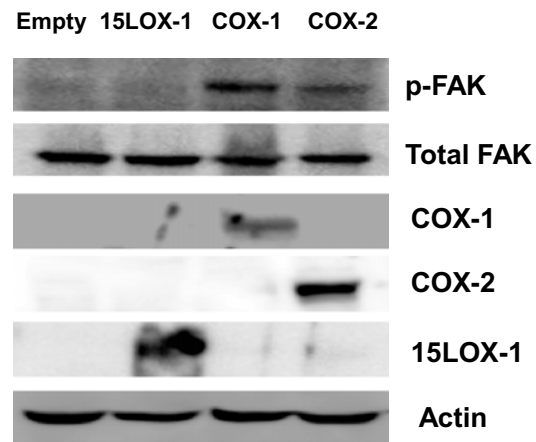

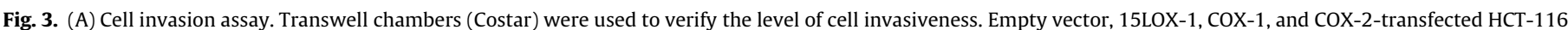

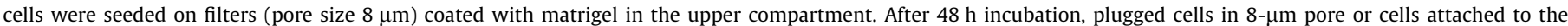

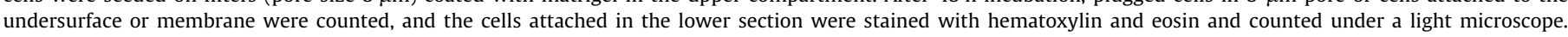

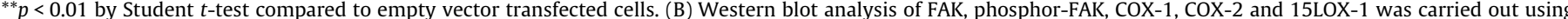

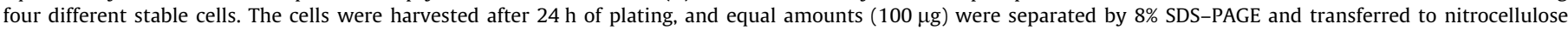
membranes. Bands corresponding to each were detected by enhanced chemiluminescence. Actin (Santa Cruz, CA) was used as a loading control. 
Most importantly, COX-2-transfected HCT-116 cells showed a continuous increase in both resistance and reactance. Micro-impedance fluctuations, indicating a measurement of cellular motility/ attachment, were also consistently higher in COX-transfected cells. The reactance measurements, however, more closely mimicked the resistance curves. This data suggests both COX-1 and COX-2 expression increase cell adhesion as well as motility compared to control cells, whereas 15LOX-1 expression showed a marginal increase of cell adhesion. Resistance generally increases when gaps between cells (spreading) and/or between cells and extracellular matrix substrates (cellular adhesion/attachment) are narrower. While there is still much discussion over the exact relationship between cell adhesion and cell impedance, these results show that COX-2-transfected HCT-116 cells have the strongest cellular adhesion of cell-to-cell and cell-to-substrate attachments.

HCT-116 cells gradually lose their strong contacts once they pass a certain time point after they reach confluences. Our micro-impedance measurements have shown these kinds of trends in several previously published papers [10,11]. As shown in Fig. 2, both empty vector and 15-LOX1 over-expressing cells had gradually decreased resistance and reactance toward day 4.5 . Thus, the peaks of those two cell lines at day 4.5 are moved to the right compared to those at day 3 (Fig. 1C).

\section{Cell invasion assay and protein expression}

To translate the opto-electric data from a real-time point to fixed-time biological assay, cell invasion assays were performed. Both COXs and 15-LOX1 cells showed increases of cell invasion activity (Fig. 3A). Interestingly, COX-2 and 15LOX-1 cells showed notably enhanced cell invasion activity, compared to COX-1 cells. The role of COXs and LOXs (particularly 12-LOX) in the regulation of surface integrin expression has been previously reported [1]. Since integrins have also been implicated in cancer development, invasion activities may be mediated by integrin expression. FAK plays an important role in cell migration and invasion. When we examined phosphorylation of FAK (Fig. 3B), both COX-1 and COX-2 cells showed increases of FAK phosphorylation at the tyrosine 576/577 site, consistent with the previous data indicating that both COX expressing cells showed cell adhesion activity as assessed by opto-electric methodology. However, little difference in phosphor-FAK expression was observed in COX-1 and COX-2 cells, as COX-2 expressing cells showed a unique pattern in opto-electric measurement.

\section{Conclusion}

Results from the measured micro-impedance and digitally processed PGL of IRCM images, as well as optical imaging analysis, delineate the quantitative and comprehensive nature of cellular adhesion and spreading to the electrode substrate, something which has not been dynamically examined in this way before. The results of this study show that the opto-electric method, using microscopic optical imaging and micro-impedance measurement, provides a quick, reliable, and comprehensive way of understanding cellular physiology including cell-cell adhesion, cell-substrate adhesion, and cellular membrane properties in real-time.

\section{Acknowledgments}

We thank Ms. Misty R. Bailey (University of Tennessee) for her critical reading of the manuscript. This work was supported by the American Cancer Society (CNE-111611), National Institutes of Health (R01CA108975), and the Center of Excellence in Livestock Diseases and Human Health, University of Tennessee, to S.J.B.

\section{References}

[1] A. Greenhough, H.J.M. Smartt, A.E. Moore, H.R. Roberts, A.C. Williams, C. Paraskeva, A. Kaidi, The COX-2/PGE2 pathway: key roles in the hallmarks of cancer and adaptation to the tumour microenvironment, Carcinogenesis 30 (2009) 377-386.

[2] P.A. Nony, S.B. Kennett, W.C. Glasgow, K. Olden, J.D. Roberts, 15(S)lipoxygenase-2 mediates Arachidonic acid-stimulated adhesion of human breast carcinoma cells through the activation of TAK1, MKK6, and p38 MAPK, J. Biol. Chem. (2005) M500418200.

[3] M. Tsujii, R.N. DuBois, Alterations in cellular adhesion and apoptosis in epithelial cells overexpressing prostaglandin endoperoxide synthase 2, Cell 83 (1995) 493-501.

[4] D.T. Bolick, A.W. Orr, A. Whetzel, S. Srinivasan, M.E. Hatley, M.A. Schwartz, C.C. Hedrick, 12/15-Lipoxygenase regulates intercellular adhesion molecule-1 expression and monocyte adhesion to endothelium through activation of RhoA and nuclear factor-\{kappa\}B, Arterioscler. Thromb. Vasc. Biol. 25 (2005) 2301-2307.

[5] N.D. Gallant, K.E. Michael, A.J. Garcia, Cell adhesion strengthening: contributions of adhesive area, integrin binding, and focal adhesion assembly, Mol. Biol. Cell 16 (2005) 4329-4340.

[6] A. Androulidaki, E. Dermitzaki, M. Venihaki, E. Karagianni, O. Rassouli, E. Andreakou, C. Stournaras, A.N. Margioris, C. Tsatsanis, Corticotropin releasing factor promotes breast cancer cell motility and invasiveness, Mol. Cancer 8 (2009) 30.

[7] R.I. Viji, V.B. Sameer Kumar, M.S. Kiran, P.R. Sudhakaran, Modulation of cyclooxygenase in endothelial cells by fibronect: relevance to angiogenesis, J. Cell. Biochem. 105 (2008) 158-166.

[8] C.K. Choi, K.D. Kihm, A.E. English, Optoelectric biosensor using indium-tinoxide electrodes, Opt. Lett. 32 (2007) 1405-1407.

[9] C.K. Choi, A.E. English, S.I. Jun, K.D. Kihm, P.D. Rack, An endothelial cell compatible biosensor fabricated using optically thin indium tin oxide silicon nitride electrodes, Biosens. Bioelectron. 22 (2007) 2585-2590.

[10] S.-H. Lee, J.H. Bahn, C.K. Choi, N.C. Whitlock, A.E. English, S. Safe, S.J. Baek, ESE$1 /$ EGR-1 pathway plays a role in tolfenamic acid-induced apoptosis in colorectal cancer cells, Mol. Cancer Ther. 7 (2008) 3739-3750.

[11] G. Park, C.K. Choi, A.E. English, T.E. Sparer, Electrical impedance measurements predict cellular transformation, Cell Biol. Int. 33 (2009) 429-433.

[12] S.J. Baek, L.C. Wilson, C.-H. Lee, T.E. Eling, Dual function of nonsteroidal antiinflammatory drugs (NSAIDs): inhibition of cyclooxygenase and induction of NSAID-activated gene, J. Pharmacol. Exp. Ther. 301 (2002) 1126-1131.

[13] J.-S. Kim, S.J. Baek, F.G. Bottone, T. Sali, T.E. Eling, Overexpression of 15lipoxygenase-1 induces growth arrest through phosphorylation of p53 in human colorectal cancer cells, Mol. Cancer Res. 3 (2005) 511-517.

[14] I. Giaever, C.R. Keese, Micromotion of mammalian cells measured electrically, Proc. Natl. Acad. Sci. USA 88 (1991) 7896-7900. 